\title{
A Clinical Outcome-Based Prospective Study on Venous Thromboembolism After Cancer Surgery
}

\author{
The @RISTOS Project
}

\author{
Giancarlo Agnelli, MD, * Giorgio Bolis, MD, $\uparrow$ Lorenzo Capussotti, MD, + \\ Roberto Mario Scarpa, MD, § Francesco Tonelli, MD,\| Erminio Bonizzoni, PhD, $\boldsymbol{\emptyset}$ Marco Moia, MD, t+ \\ Fabio Parazzini, MD, † Romina Rossi, MD, * Francesco Sonaglia, MD, * Bettina Valarani, PhD, ** \\ Carlo Bianchini, MD, †† and Gualberto Gussoni, MD, PhD††
}

Summary Background Data: The epidemiology of venous thromboembolism (VTE) after cancer surgery is based on clinical trials on VTE prophylaxis that used venography to screen deep vein thrombosis (DVT). However, the clinical relevance of asymptomatic venography-detected DVT is unclear, and the population of these clinical trials is not necessarily representative of the overall cancer surgery population.

Objective: The aim of this study was to evaluate the incidence of clinically overt VTE in a wide spectrum of consecutive patients undergoing surgery for cancer and to identify risk factors for VTE. Methods:@RISTOS was a prospective observational study in patients undergoing general, urologic, or gynecologic surgery. Patients were assessed for clinically overt VTE occurring up to $30 \pm$ 5 days after surgery or more if the hospital stay was longer than 35 days. All outcome events were evaluated by an independent Adjudication Committee.

Results: A total of 2373 patients were included in the study: 1238 (52\%) undergoing general, $685(29 \%)$ urologic, and $450(19 \%)$ gynecologic surgery. In-hospital prophylaxis was given in $81.6 \%$ and postdischarge prophylaxis in $30.7 \%$ of the patients. Fifty patients $(2.1 \%)$ were adjudicated as affected by clinically overt VTE (DVT, $0.42 \%$; nonfatal pulmonary embolism, $0.88 \%$; death $0.80 \%$ ). The incidence of VTE was $2.83 \%$ in general surgery, $2.0 \%$ in gynecologic surgery, and $0.87 \%$ in urologic surgery. Forty percent of the events occurred later than 21 days from surgery. The overall

From the *Division of Internal and Cardiovascular Medicine, University of Perugia, Perugia, Italy; †Obstetrics and Gynecology Clinic I, University of Milan, Milan, Italy; $\ddagger$ Department of Surgical Oncology, Istituto per la Ricerca e la Cura del Cancro, Candiolo, Turin, Italy; §Department of Urology, "San Luigi" Hospital, Orbassano, Turin, Italy; |Department of Clinical Physiopathology, University of Florence, Florence, Italy; $\uparrow$ Institute of Medical Statistics and Biometry, University of Milan, Milan, Italy; $\$$ Angelo Bianchi Bonomi Hemophilia and Thrombosis Center, IRCCS Maggiore Hospital, Milan, Italy; **Hyperphar Group, Milan, Italy; and $\dagger \uparrow$ Scientific Department, Italfarmaco, Milan, Italy.

Supported by a grant from Italfarmaco S.p.A., Milan, Italy.

Reprints: Giancarlo Agnelli, MD, Sezione di Medicina Interna e Cardiovascolare, Dipartimento di Medicina Interna, Università di Perugia, Via Enrico Dal Pozzo, 06123 Perugia, Italy. E-mail: agnellig@unipg.it.

Copyright (C) 2005 by Lippincott Williams \& Wilkins

ISSN: 0003-4932/06/24301-0089

DOI: $10.1097 / 01$. sla.0000193959.44677.48 death rate was $1.72 \%$; in $46.3 \%$ of the cases, death was caused by VTE. In a multivariable analysis, 5 risk factors were identified: age above 60 years $(2.63,95 \%$ confidence interval, $1.21-5.71)$, previous VTE $(5.98,2.13-16.80)$, advanced cancer $(2.68,1.37-5.24)$, anesthesia lasting more than 2 hours $(4.50,1.06-19.04)$, and bed rest longer than 3 days $(4.37,2.45-7.78)$.

Conclusions: VTE remains a common complication of cancer surgery, with a remarkable proportion of events occurring late after surgery. In patients undergoing cancer surgery, VTE is the most common cause of death at 30 days after surgery.

(Ann Surg 2006;243: 89-95)

V enous thromboembolism (VTE) is a common complication in patients undergoing surgery. ${ }^{1,2}$ Cancer surgery seems to have at least twice the risk of postoperative deep vein thrombosis (DVT) and more than 3 times the risk of fatal pulmonary embolism (PE) than similar procedures in noncancer patients. $^{3-5}$

The epidemiology of VTE after cancer surgery is essentially based on the findings of intervention clinical trials on VTE prophylaxis that used venography to detect DVT. ${ }^{6}$ However, the clinical relevance of asymptomatic venography-detected DVT is unclear, ${ }^{7}$ and patients included in these clinical trials are not necessarily representative of the overall cancer surgery population since in these studies strict inclusion and exclusion criteria are generally used.

Current epidemiology of VTE after cancer surgery is mainly based on studies performed over 20 years ago. ${ }^{5}$ The risk for thromboembolic complications in cancer surgery may have altered over the years as this particular surgery has witnessed a number of recent changes. On one hand, improvements in surgical techniques, more prompt mobilization, improved use of prophylaxis, and advances in perioperative care may have reduced the risk for VTE. On the other hand, more extensive procedures in older and sicker patients and preoperative chemotherapy and/or radiotherapy may have increased the risk of VTE in patients undergoing cancer surgery nowadays. Furthermore, shorter hospital stays for 
surgery plus extended duration of prophylaxis could influence the time course of the occurrence of thromboembolic events today. Finally, most of the available data have been obtained from studies that limited their observation to the first 7 to 10 postoperative days, ${ }^{5}$ while few data have been reported on the incidence of clinically overt events over longer periods. ${ }^{8}$

All these elements highlight the need for up-to-date data on the epidemiology of VTE following cancer surgery.

The aim of this study was to prospectively evaluate the incidence of clinically overt VTE in a wide spectrum of consecutive patients undergoing cancer surgery and to identify potential risk factors for VTE. Patients were assessed for clinically overt VTE occurring up to $30 \pm 5$ days from surgery or more in the case of longer hospital stays.

\section{MATERIALS AND METHODS}

@RISTOS was a prospective registry performed in 31 Italian surgery departments with a high rate of cancer operations. Data were collected from consecutive patients undergoing abdominal, thoracic, gynecologic, and urologic surgery for cancer. Both curative and palliative, nonendoscopic or endoscopic interventions, interventions on primary tumor(s) and/or secondary lesion(s) were included.

The aim of the study was to assess the incidence of clinically overt VTE (DVT, PE, death due to PE) in cancer patients during a period of $30 \pm 5$ days from surgery or more if hospital stay was longer than 35 days, and to identify potential risk factors for VTE.

At hospital admission information on patients' characteristics (medical history for possible risk factors for VTE and concomitant diseases) and presurgery anticancer treatments were collected. At discharge, relevant data were recorded concerning surgery, antithrombotic prophylaxis, postsurgery anticancer treatments, bleeding complications, and venous thromboembolic events. At $30 \pm 5$ days from surgery, information on antithrombotic prophylaxis, adjuvant chemotherapy/ radiotherapy, bleeding complications, and venous thromboembolic events were collected.

The registry did not issue diagnostic algorithms or guidelines for management of patients included in the study. Patients were instructed to report to the study center if symptoms developed that were suggestive of VTE or bleeding.

All clinically suspected venous thromboembolic events and all cases of death were reviewed by an external independent Adjudication Committee composed of 3 clinicians. Two reviewed independently the patients' clinical documents (hospital chart, imaging examinations such as ultrasonography, venography, perfusion lung scan, CT scan, and autopsy findings, if available). In the case of divergent interpretation, the patients' records were reviewed by the third member.

The study was approved by the Ethic Committees of all participating centers. Informed consent for handling of personal data was obtained from all patients. Data collection and management were performed by using a Web-based system (Hypernet, Hyperphar Group, Milan, Italy, with CINECA technology, Bologna, Italy), allowing real-time control of collected data. A minimum of 3 monitoring visits was performed in each center to verify the consistency between source documents and data reported on the electronic case report forms.

\section{Statistical Analysis}

A sample size of 2000 patients was considered appropriate to document a reliable estimate of the incidence of clinically overt VTE with sufficiently narrow confidence intervals of the estimate, by hypothesizing an incidence between $2 \%$ and $5 \%$.

Summary statistics were calculated for all patient variables. For continuous variables, mean, standard deviation (SD), median, minimum, and maximum values were assessed.

Statistical analysis was carried out using SAS version. 8.2. Data analysis was carried out according to a pre-established plan. The association with the study outcomes of prognostic factors, such as age, prior DVT or PE, tumor stage, duration of anesthesia, duration of bed rest, was evaluated by a multivariable logistic regression. Covariates for logistic regression analysis were selected on the basis of their clinical plausibility and following the general rule of thumb, which states that the ratio between the overall number of events and the number of explanatory variables should be at least equal to 10 . An additional multivariable logistic regression analysis was performed to relate the use or not of antithrombotic prophylaxis with some covariates (age, tumor staging, type and duration of anesthesia, endoscopic or nonendoscopic surgery, intervention on primary tumor(s) and/or secondary lesion(s)).

Odds ratios (ORs) and 95\% confidence intervals (CI) were reported with 2 -tailed probability values. A $P$ value $\leq 0.05$ was considered statistically significant.

\section{RESULTS}

\section{Study Population and Surgery}

A total of 2373 patients were included in the study. Male patients were $1283(54.1 \%)$; the mean age of study population was 63.6 years (SD, 12.4 years). A history of VTE was reported by $41(1.7 \%)$ patients. A total of 107 patients $(4.5 \%)$ underwent preoperative chemotherapy and/or radiotherapy. Major surgery had been performed in $2.8 \%$ of patients in the 3 months before inclusion in the study.

A total of 1238 patients $(52.2 \%)$ underwent general surgery (49.1\% abdominal and 3.1\% thoracic surgery), and $450(18.9 \%)$ and $685(28.8 \%)$ patients had gynecologic and urologic surgery, respectively. Details on the characteristics of interventions are specified in Table 1 . In the majority of cases, primary tumor was the target $(77.4 \%)$ and radicality (ablation of cancer lesion(s) and of regional nodes, if the case), the intention (78.9\%) of surgery.

Endoscopic surgery was performed in $21.2 \%$ of patients, most of them in urology (61\% of this specific setting). Duration of anesthesia was longer than 2 hours in $76.3 \%$ of patients, mainly in general surgery, while urology presented a significant proportion of interventions lasting less than 45 minutes $(32.0 \%)$.

Tumor staging was determined by means of TNM or FIGO classifications in $92.9 \%$ of patients. These patients were reviewed by experts in the specific surgical field to 


\begin{tabular}{|c|c|}
\hline Characteristic & No. (\%) of Patients \\
\hline \multicolumn{2}{|l|}{ Site(s) of surgery } \\
\hline Primary tumor & $1836(77.4)$ \\
\hline Multiple primary tumors & $32(1.3)$ \\
\hline Primary tumor(s) + metastases & $272(11.4)$ \\
\hline Metastases & $233(9.8)$ \\
\hline \multicolumn{2}{|l|}{ Type of surgery } \\
\hline Radical & $1872(78.9)$ \\
\hline Not radical & $338(14.2)$ \\
\hline Palliative & $163(6.9)$ \\
\hline \multicolumn{2}{|l|}{ Endoscopic surgery } \\
\hline Total & $504(21.2)$ \\
\hline General surgery & $32(2.6)^{*}$ \\
\hline Gynecology & $54(12.0)^{*}$ \\
\hline Urology & $418(61.0)^{*}$ \\
\hline \multicolumn{2}{|l|}{ Type of anesthesia } \\
\hline General & $2121(89.4)$ \\
\hline Local & $252(10.6)$ \\
\hline \multicolumn{2}{|l|}{ Duration of anesthesia } \\
\hline Total $<45 \mathrm{~min}$ & $227(9.6)$ \\
\hline $0.45 \mathrm{~min}-1.59 \mathrm{hr}$ & $336(14.1)$ \\
\hline$\geq 2.00 \mathrm{hr}$ & $1810(76.3)$ \\
\hline General surgery $<45$ min & $2(0.2)$ \\
\hline $0.45 \mathrm{~min}-1.59 \mathrm{hr}$ & $102(8.2)$ \\
\hline$\geq 2.00 \mathrm{hr}$ & 1134 (91.6) \\
\hline Gynecology $<45 \mathrm{~min}$ & $6(1.3)$ \\
\hline $0.45 \mathrm{~min}-1.59 \mathrm{hr}$ & $87(19.3)$ \\
\hline$\geq 2.00 \mathrm{hr}$ & $357(79.4)$ \\
\hline Urology $<45 \mathrm{~min}$ & $219(32.0)$ \\
\hline $0.45 \mathrm{~min}-1.59 \mathrm{hr}$ & $147(21.4)$ \\
\hline$\geq 2.00 \mathrm{hr}$ & $319(46.6)$ \\
\hline
\end{tabular}

categorize the case as "advanced" or "not advanced": $45.9 \%$ and $47.0 \%$ of patients were classified as affected with "notadvanced" or "advanced" disease, respectively. The more frequent known sites of primary tumor (frequency $\geq 50$ cases) are listed in Table 2.

\section{TABLE 2. Site of Primary Tumor}

\begin{tabular}{lc}
\hline Site of Primary Tumor & No. (\%) \\
\hline Urinary bladder & $447(18.8)$ \\
Colon & $362(15.3)$ \\
Rectum & $209(8.8)$ \\
Uterus & $201(8.4)$ \\
Stomach & $179(7.5)$ \\
Prostate & $140(5.9)$ \\
Ovary & $139(5.9)$ \\
Kidney & $79(3.3)$ \\
Liver & $71(3.0)$ \\
Pancreas & $64(2.7)$ \\
Lung & $57(2.4)$ \\
\hline
\end{tabular}

The mean duration ( \pm SD) of the postoperative hospital stay was $12.3( \pm 8.7), 9.1( \pm 6.9)$, and $6.2( \pm 5.6)$ days after general, gynecologic, and urologic surgery, respectively. Bed rest following intervention was shorter than 4 days in the majority of patients $(84.4 \%)$, with no significant differences among the 3 types of surgery. Postoperative chemotherapy and/or radiotherapy were reported in $21.5 \%$ of the patients.

\section{Antithrombotic Prophylaxis}

In-hospital antithrombotic prophylaxis was given in $81.7 \%$ of patients (general surgery, $86.8 \%$; gynecology, $82.7 \%$; urology, $71.7 \%$ ), while postdischarge prophylaxis was used in $30.7 \%$ of the patients (general surgery, $30.1 \%$; gynecology, 29.5\%; urology, 32.5\%). A prophylaxis lasting more than 21 days was used in 553 patients $(23.3 \%)$.

Low molecular weight heparin (LMWH) was used in approximately three fourths of the treated patients, both for in-hospital and postdischarge prophylaxis (74.3\% and $77.2 \%$ of cases, respectively). Specifically, LMWH prophylaxis was started before surgery in $71.1 \%$ of the cases. Physical methods, mainly as combination therapy, were used in $10.4 \%$ and $5.5 \%$ of patients undergoing prophylaxis during hospital stay or following discharge, respectively. Other pharmacologic methods of prophylaxis were unfractionated heparin $(18.7 \%$ and $3.9 \%$ of patients with in-hospital or postdischarge prophylaxis) and heparinoids (2.6\%, in-hospital). Antiplatelets and oral anticoagulants, $10.9 \%$ and $3.9 \%$, respectively, were given postdischarge in patients that had temporarily interrupted these agents before surgery.

\section{Venous Thromboembolic Events}

A total of $2360(99.5 \%)$ patients were followed up as stated by study protocol. A total of 92 patients with suspected thromboembolic events or deaths were reported during the study and reviewed by the external independent Adjudication Committee.

Fifty of these patients $(2.1 \%)$ were adjudicated as affected by VTE or as dead due to PE (Fig. 1A). Isolated DVT was confirmed in $10(0.40 \%)$ patients, nonfatal PE in 21 $(0.88 \%)$ patients (in 16 cases isolated PE, in 5 associated with DVT). Death was attributed to VTE in 19 cases $(0.82 \%)$. The incidence of VTE was $2.8 \%$ in general surgery, $2.0 \%$ in gynecologic surgery, and $0.87 \%$ in urologic surgery (Fig. 1B). Regarding thromboembolic events, the clinical interpretation of the local investigators and the Adjudication Committee was consistent in $85 \%$ of cases. In the 8 patients with an inconsistent interpretation, the Adjudication Committee called VTE 6 cases not locally diagnosed as DVT or PE, while 2 episodes locally adjudicated as VTE were not confirmed by the external Committee.

For primary tumor sites present in more than 50 patients, the rate of patients with VTE is shown in Figure 2. The rate of thromboembolic events in patients with gastroenteric tract, lung, or kidney cancer was higher than the overall incidence rate. No patients with primary hepatic tumor experienced VTE; however, in 5 patients with VTE, an intervention involving liver (for secondary lesions) had been done.

Table 3 shows the distribution of patients with and without VTE according to selected covariates and the results 


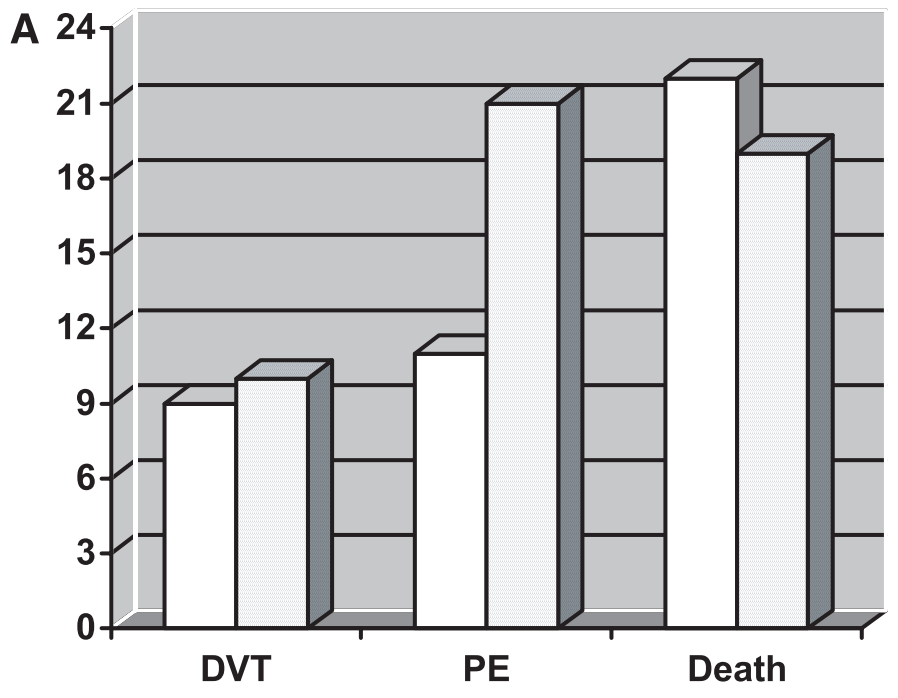

\section{$\square$ Not confirmed ( $n=42) \square$ Confirmed $(n=50)$}

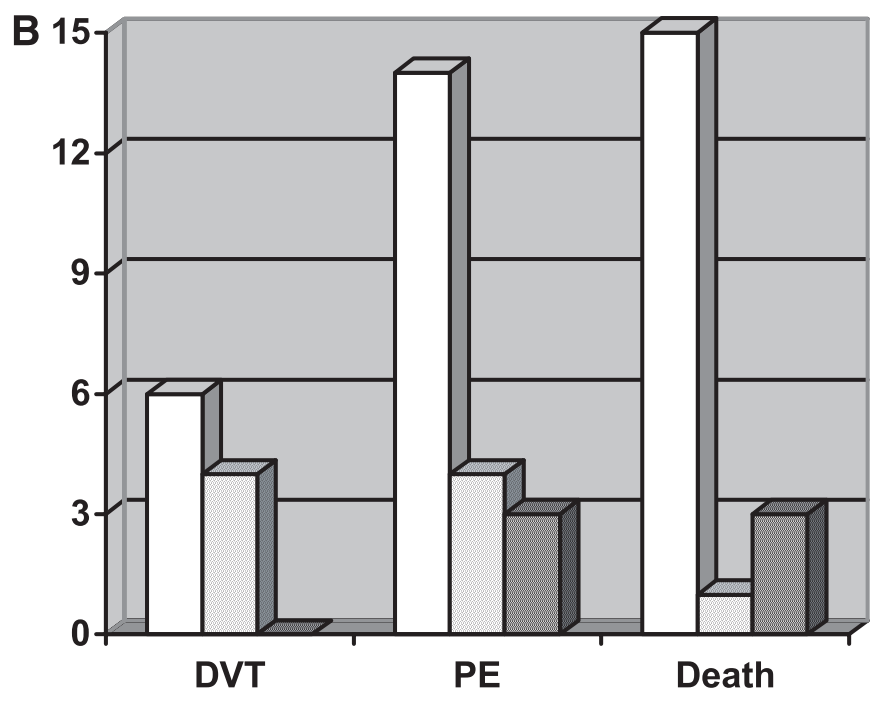

$\square$ Gen. surg. $\square$ Gynecology $\square$ Urology

FIGURE 1. A, Venous thromboembolic events confirmed or not by the Adjudication Committee. B, Distribution of confirmed events by surgery.

of a multivariable logistic regression analysis performed with the aim to identify potential risk factors for VTE. The risk of VTE increased with age: in comparison with patients under 60 years, the OR of VTE was 2.6 (95\% CI, 1.2-5.7) in those over 60 years. Likewise, a previous history of VTE (OR, 6.0; 95\% CI, 2.1-16.8), a prolonged duration of surgical procedure (anesthesia lasting over 2 hours versus less than 2 hours; OR, 4.5; 95\% CI, 1.1-19.0), a prolonged bed stay after

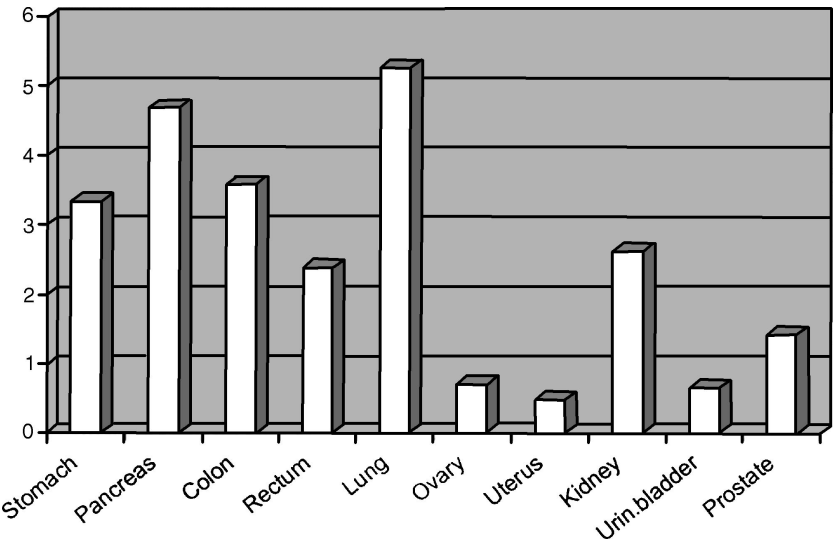

FIGURE 2. Incidence of VTE in reference to site of primary tumor.

surgery (more than or equal to 4 days versus less than 4 days; OR, 2.7; 95\% CI, 1.4-5.2), and an advanced tumor (OR, 4.4; 95\% CI, 2.5-7.8) all appeared to enhance the risk of VTE.

Venous thromboembolic events occurred during prophylaxis in the majority of cases (37, corresponding to $74 \%$ ). In 12 VTE patients (24\%), prophylaxis had been discontinued (1-23 days) before the occurrence of the event; in the remaining patient, antithrombotic prophylaxis was not used at all.

An additional multivariable logistic regression analysis was performed to evaluate the factors which influence the surgeon's decision to use or not antithrombotic prophylaxis. Some factors were significantly associated with the use of prophylaxis: age 60 years or older versus under 60 years (OR, $1.5 ; 95 \% \mathrm{CI}, 1.2-1.9)$, general versus local anesthesia (OR, $2.3 ; 1.6-3.3)$, scheduled duration of anesthesia longer than or equal to 2 hours versus less than 2 hours (OR, 3.7; 2.8-5.0), advanced versus not advanced staging of the disease (OR, $1.5 ; 1.1-1.9)$, combined surgery of primary tumor and metastasis versus intervention on primary tumor alone (OR, 2.4; 1.3-4.2). Prophylaxis was used less frequently when surgery was performed for secondary lesions in comparison to primary tumors (OR, 0.6; 0.4-0.8). There

TABLE 3. Prognostic Risk Factors for VTE: Multivariable Logistic Regression Analysis

\begin{tabular}{|c|c|c|c|c|}
\hline Variable & Effect & $\begin{array}{l}\text { No. of Patients } \\
\text { VTE/Non-VTE }\end{array}$ & OR & $95 \%$ CI \\
\hline Age class & $\begin{array}{l}\geq 60 \text { versus } \\
\quad<60 \mathrm{yr}\end{array}$ & $\begin{array}{l}\geq 60 \text { yr: } 42 / 1.516 \\
<60 \text { yr: } 8 / 807\end{array}$ & 2.6 & $1.2-5.7$ \\
\hline Previous VTE & Yes versus no & $\begin{array}{l}\text { Yes: } 5 / 36 \\
\text { No: } 45 / 2.287\end{array}$ & 6.0 & $2.1-16.8$ \\
\hline Anesthesia & $\begin{array}{l}\geq 2: 00 \text { versus } \\
\quad<2: 00 \mathrm{hr}\end{array}$ & $\begin{array}{l}\geq 2: 00 \mathrm{hr}: 48 / 1.762 \\
<2: 00 \mathrm{hr}: 2 / 561\end{array}$ & 4.5 & $1.1-19.0$ \\
\hline Staging & $\begin{array}{c}\text { Advanced versus } \\
\text { not advanced }\end{array}$ & $\begin{array}{l}\text { Advanced: } \\
38 / 1.078 \\
\text { Not advanced: } \\
12 / 1.245\end{array}$ & 2.7 & $1.4-5.2$ \\
\hline Bed rest & $\begin{array}{l}\geq 4 \text { versus } \\
\quad<4 \text { days }\end{array}$ & $\begin{array}{l}\geq 4 \text { days: } 25 / 346 \\
<4 \text { days: } 25 / 1.977\end{array}$ & 4.4 & $2.5-7.8$ \\
\hline
\end{tabular}




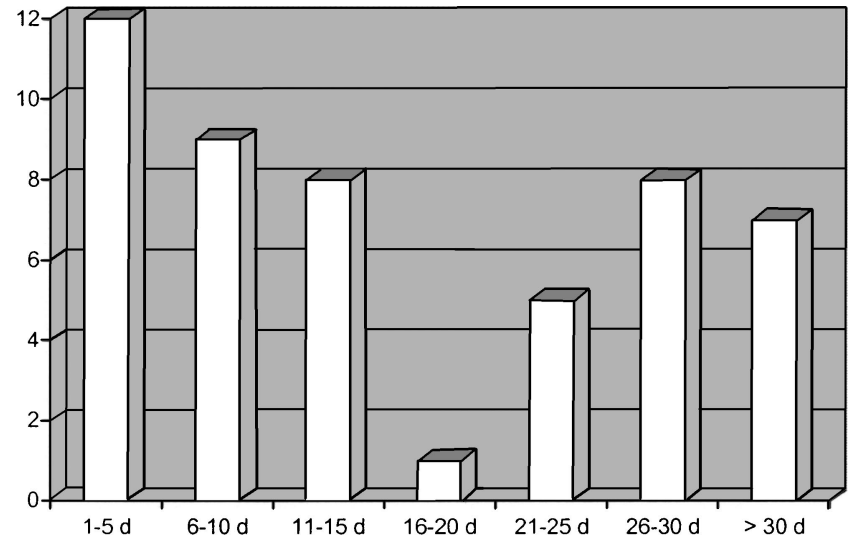

FIGURE 3. Time distribution of venous thromboembolic events.

was no significant difference in the physician's decision regarding nonendoscopic versus endoscopic surgery (OR, $0.9 ; 0.7-1.4)$.

Time distribution of venous thromboembolic events is shown in Figure 3. Mean $( \pm$ SD) time of occurrence of thromboembolic events was $17.2 \pm 14.0$ days (range, $2-58$ days). Forty percent of the events (20 cases) were observed more than 21 days from surgery; in 9 of these patients (45\%), the event occurred after prophylaxis was withdrawn. The risk factors listed in Table 3 were analyzed separately for early (ie, within 15 days from surgery) or late VTE: no difference in the OR estimates emerged (data not shown).

\section{Bleeding}

Bleeding episodes were recorded in $9.9 \%$ of patients. In half of the bleeding episodes (5.0\%), blood transfusion was not required. When transfusions were necessary, surgeons were asked to classify the episodes as "expected" or "unexpected" according to the type of surgery and the patient's condition. In the majority of cases $(69.7 \%)$, bleeding was considered "expected." The incidence of unexpected bleeding requiring transfusion was $1.5 \%$. In 3 of 4 patients who died due to bleeding, prophylaxis had been discontinued when bleedings occurred.

Bleeding occurred more frequently in patients who received antithrombotic prophylaxis in comparison to patients not treated $(11.4 \%$ versus $3.0 \%, P<0.001)$. By means of a multivariable logistic regression analysis, we found that antithrombotic prophylaxis was given more frequently in patients aged more than 60 years, in the case of surgery lasting more than 2 hours, and in patients with advanced disease.

\section{Mortality}

Forty-one patients died during the scheduled observation period (overall death rate, $1.72 \%$ ). Death rates in the specific surgical settings were $2.91 \%$ for general surgery, $0.22 \%$ for gynecology surgery, and $0.58 \%$ for urology. Venous thromboembolism was adjudicated as the most common cause of death (19 of 41 cases, $46.3 \%$ ). The other causes of death were disease progression ( 5 cases), liver failure (3 cases), bleeding ( 3 cases), stroke ( 2 cases), sepsis ( 2 cases), and cardiac arrest, acute myocardial infarction, disseminated intravascular coagulation, pancreatitis, respiratory failure, renal failure, and multiorgan failure (1 case each). In the 4 bleeding-related deaths, pharmacologic antithrombotic prophylaxis was ongoing at the time of the event in 1 patient, it had been previously discontinued (16 and 33 days before) in 2 , while it had not been used at all in the remaining one (hemorrhagic stroke).

\section{DISCUSSION}

In recent years, cancer surgery has undergone a number of changes; hence, the need for up-to-date data on the epidemiology of VTE in this clinical setting. This study shows that the incidence of venous thromboembolic events after cancer surgery is not negligible. The risk of VTE persists after the conventional period of antithrombotic prophylaxis. Risk factors, seen in previous venography trials, have been confirmed in this clinical outcome-based study.

The clinical relevance of asymptomatic venographydetected DVT has been challenged over the years. ${ }^{7}$ This study, focused on clinically overt venous thromboembolic events, confirms the clinical importance of postoperative VTE after cancer surgery. It is noteworthy that most of the events were PE and death. The relatively low incidence of DVT is potentially explained by the intrinsic nature of postoperative DVT: nonocclusive and therefore often asymptomatic. Our study seems to indicate that nonocclusive asymptomatic DVT leads to PE. A potential, although partial, impact of asymptomatic pelvic DVT in this context cannot be excluded; in our study, 14 of 50 cases of total thromboembolic events and 6 of 20 cases with isolated symptomatic PE occurred in patients undergoing pelvic surgery.

In recent years, prolonged prophylaxis has been proposed in cancer surgery on the base of a venography study. ${ }^{8}$ Our study confirms previous findings on the persistence of risk of VTE after the perioperative period, showing that $40 \%$ of VTE events occurred more than 21 days from surgery. Interestingly, antithrombotic prophylaxis was extended beyond 3 weeks after surgery in only $23 \%$ of patients, and almost half of the VTE events occurring later than 21 days took place after withdrawal of prophylaxis.

In this study, age, previous VTE, advanced disease, duration of surgery, and bed rest are confirmed as risk factors for VTE. These features or some of these may explain the difference in the incidence observed between the individual types of surgery. Indeed, the incidence of VTE events in general surgery was 3 times higher than in urology surgery. General surgery, in comparison to urology, was characterized by a higher proportion of interventions of prolonged duration, and patients more frequently had advanced disease $(69 \%$ versus $12 \%$ ).

Our study was not designed to address the issue of bleeding complications after cancer surgery as well as to evaluate the benefit to risk ratio of antithrombotic prophylaxis in this clinical setting. However, we observed a high overall bleeding rate as well as a higher bleeding rate in patients 
receiving prophylaxis in comparison to those not receiving prophylaxis.

The overall high bleeding rate observed in our study could be explained by the fact that surgeons were asked to report all bleeding events regardless of whether they were clinically major or minor. Of interest, about $50 \%$ of the reported bleeding events did not require blood transfusions as they were probably minor bleedings. In addition, the high bleeding rate observed in our study could be explained by the intrinsic differences between the study populations of registries and clinical trials. Patients included in clinical trials are usually selected by strict criteria and this often results in the exclusion of patients at high bleeding risk.

The higher bleeding rate seen in patients receiving prophylaxis in comparison to those not receiving prophylaxis could have several explanations. Reporting of intraoperative or postoperative bleedings was mainly based on clinical perception; therefore, the open design of our study may have led to an overestimation of the bleeding complications in patients receiving antithrombotic prophylaxis, as it was observed previously. ${ }^{9}$ Antithrombotic prophylaxis was given more frequently in patients aged more than 60 years, in the case of surgery lasting more than 2 hours, and in patients with advanced disease. Therefore, prophylaxis was probably given to patients at high risk of bleeding. However, the rate of unexpected bleeding requiring transfusion was as low as $1.5 \%$. Based on all these considerations, it is conceivable to believe that the increased bleeding rate seen in our registry do not challenge the documented favorable benefit to risk ratio of antithrombotic prophylaxis in cancer surgery.

Our registry, like other observational studies, has certainly some intrinsic limitations. Among these, the absence of standardization in the prophylaxis regimens. Nevertheless, it should be mentioned that, consistent with the current guidelines, most of the patients received LMWH prophylaxis.

However, our study has some methodologic strengths that may make our results valid. We included consecutive patients undergoing a wide spectrum of cancer surgery. Patients were closely followed up as scheduled by study protocol in $99 \%$ of the cases. All suspected clinical events were prospectively collected and validated by an external independent Adjudication Committee using predefined standard criteria. Further, death accounted for a large proportion of the outcome events. As quality assurance of recorded data, the study included active monitoring in each study center to verify the consistency between source documents and data reported on electronic case report forms. Moreover, electronic data capture allowed a central, real-time check of recorded data.

\section{CONCLUSION}

The results of our study have potential implications for clinical practice and future research. The persistently high incidence of VTE after cancer surgery, despite improvements in patient care, requires a high level of attention even nowadays. The incidence of VTE events remains high despite prophylaxis, hence requiring further improvement, particu- larly in patients with known risk factors. The high proportion of late occurring events suggests the need of extending antithrombotic prophylaxis beyond hospital discharge and/or the conventional perioperative period.

\section{ACKNOWLEDGMENTS}

The authors thank Marco Sardina for his contribution in the design of the study, Eva Tikotin for reviewing the manuscript, Dina Casiraghi and Roberta Starnini for their secretarial assistance, the nursing staff of the participating centers for their enthusiastic cooperation, and the patients included in the study for their trust and support.

Contributors: G. Agnelli coordinated the study. G. Bolis, L. Capussotti, R. M. Scarpa, F. Tonelli, M. Moia, R. Rossi, F. Sonaglia, B. Valarani, C. Bianchini, and G. Gussoni took part in the planning and execution of the study. G. Agnelli, E. Bonizzoni, F. Parazzini, R. Rossi, and G. Gussoni wrote the paper. E. Bonizzoni was responsible for the statistical analysis. All authors reviewed the paper. The Adjudication Committee members were M. Moia, P. Bucciarelli, and I. Martinelli (Angelo Bianchi Bonomi Hemophilia and Thrombosis Center, IRCCS Maggiore Hospital, Milan).

Members of the@RISTOS Study Groupwere the following: European Institute of Oncology, Division of General Surgery, Milano (B. Andreoni, R. Biffi, S. Cenciarelli); Department of Surgical Oncology, Istituto per la Ricerca e la Cura del Cancro, Candiolo, Torino (L. Capussotti, M. Calgaro, R. Polastri, D. Zorzi); Department of Urology, "G. Mazzini" Hospital, Teramo, Chair of Urology, L'Aquila University School of Medicine, L'Aquila (A. Tubaro, R. Perna, C. Vicentini); General Surgery, Cancer Institute, Bari (S. Montemurro, C. Caliandro, E. Ruggeri); General Surgery, "Humanitas" Institute, Rozzano (L. Gennari, A. Brocchi, V. Quagliuolo); Department of Urology, "San Luigi" Hospital, Orbassano (R. M. Scarpa, F. Ragni); Department of Urology and Andrology, "St. Anna" Hospital, Como (G. Conti, E. Cretarola); Department of Urology II, University of Bari (A. Pagliarulo, G. D'Achille); General and Oncologic Surgery, University of Perugia (A. Bartoli, C. Bussotti, E. Ricci, A. Servoli); Department of Urology, "Madonna delle Grazie" Hospital, Matera (G. Carrieri, T. Corvasce, G. Disabato); Division of General Surgery I, "Careggi” Hospital, Firenze (R. Moretti, L. Bencini, S. Cantafio, M. Scatizzi); Department of Gynecology Oncology, Catholic University, Roma (G. Scambia, E. Foti); Gynecology Department, Ospedali Riuniti, Bergamo (L. Frigerio, L. Carlini, A. Gallo); Urology Department, "Federico II" University, Napoli (V. Mirone, F. Mangiapia, A. Palmieri); Department of Surgery and Blood Bank, National Cancer Institute, Milano (V. Mazzaferro, F. Ravagnani, R. Romito); Department of Obstetrics and Gynecology, S. Raffaele Hospital, Milano (A. Ferrari, G. Mangili, P. Carnelli, R. Viganò); General Surgery, Insubria University of Insubria, Varese (R. Dionigi, P. Castelli, R. Caronno, G. Piffaretti, L. Uccella); National Cancer Institute, "Fondazione Pascale," Napoli (V. Parisi, F. Ruffolo, C. Verrusio); Department of Gynecology and Obstetrics, IRCCS 
Policlinico San Matteo, Pavia (S. Tateo, L. Mereu, S. Salamano); Department of Clinical Physiopathology, University of Firenze ( $F$. Tonelli, R. Valanzano); Institute of General Surgery II, University of Padova (M. Lise, D. Nitti, C. Belluco, M. Forlin); Oncology Surgery Department, National Cancer Institute, Aviano (C. Rossi, R. Massarut); Department of Urology, "S.S. Trinità" Hospital, University of Cagliari (E. Usai, A. De Lisa, P. Cirillo); Department of General, Geriatric, Oncological Surgery and Advanced Technologies, "Federico II" University, Napoli (G. Mosella, G. Quarto, A. Cappiello, F. Mosella, M. Pastore); Obstetrics and Gynecology Institute, University Bicocca, Milan, "San Gerardo dei Tintori" Hospital, Monza (C. Mangioni, A. Pellegrino, A. Villa); Thoracic Surgery, University of Perugia (G. Daddi, L. Cagini, F. Calzolari); Oncology Surgery, "A. Cardarelli" Hospital, Napoli (R. Nigro, G. Confuorto); Chair of Gynecology II, Spedali Riuniti, Brescia (S. Pecorelli, F. Odicino, T. Sacconi); Gynecology and Obstetrics Department, "Cannizzaro" Hospital, Catania (P. Scollo, G. Scibilia); Department of Surgery, University of Bologna (A. Del Gaudio, D. Accorsi); Department of Urology, Ospedale Civile, Portogruaro (G. Fiaccavento, F. Plaino).

\section{REFERENCES}

1. Kakkar VV, Howe CT, Nicolaides AN, et al. Deep vein thrombosis of the leg: is there a 'high risk' group? Am J Surg. 1970;120:527-530.

2. Walsh JJ, Bonnar J, Wright FW. A study of pulmonary embolism and deep leg vein thrombosis after major gynaecologic surgery using labelled fibrinogen phlebography and lung scanning. J Obstet Gynaecol Br Commonw. 1974;81:311-316.

3. Huber O, Bounameux H, Borst F, et al. Postoperative pulmonary embolism after hospital discharge: an underestimated risk. Arch Surg. 1992; 127:310-313.

4. Gallus AS. Prevention of post-operative deep leg vein thrombosis in patients with cancer. Thromb Haemost. 1997;78:126-132.

5. Geerts WH, Pineo JF, Heit JA, et al. Prevention of venous thromboembolism: the seventh ACCP conference on antithrombotic and thrombolytic therapy. Chest. 2004;126(suppl):338-400.

6. ENOXACAN Study Group. Efficacy and safety of enoxaparin versus unfractionated heparin for prevention of deep vein thrombosis in elective cancer surgery: a double-blind randomised multicentre trial with venographic assessment. Br J Surg. 1997;84:1099-1103.

7. Lowe GDO, Sandercock PAG, Rosendaal FR. Prevention of venous thromboembolism after orthopaedic surgery: is fondaparinux an advance? Lancet. 2003;362:504-505.

8. Bergqvist D, Agnelli G, Cohen AT, et al. Duration of prophylaxis against venous thromboembolism with enoxaparin after surgery for cancer. N Engl J Med. 2002;346:975-980.

9. Settembrini PG, Schiassi M, Olivari N, et al. Perception of bleeding risk and heparin prophylaxis. Lancet. 1990;336:1585. 